\title{
Optically Controlled Cell Discrimination and Trapping Using Optoelectronic Tweezers
}

\author{
Aaron T. Ohta, Student Member, IEEE, Pei-Yu Chiou, Huan L. Phan, Steven W. Sherwood, Joon M. Yang, \\ Aldrich N. K. Lau, Hsan-Yin Hsu, Arash Jamshidi, and Ming C. Wu, Fellow, IEEE
}

(Invited Paper)

\begin{abstract}
Optoelectronic tweezers (OET) provide a powerful tool for the manipulation of micro- and nanoparticles. The OET device produces an optically controlled dielectrophoretic force, allowing complex dynamic manipulation patterns using light intensities up to 100000 times lower than that of optical tweezers. Using OET, we demonstrate the separation of live and dead human B cells, and the separation of HeLa and Jurkat cells. We also present, for the first time, a modified single-sided OET device that promises to facilitate the integration of OET and microfluidics. Unlike standard OET, this single-sided OET device produces electric fields that are oriented parallel to the plane of the device. We demonstrate the manipulation of polystyrene beads using this new single-sided OET device, and discuss its capabilities.
\end{abstract}

Index Terms-Dielectrophoresis (DEP), optical tweezers, optically induced dielectrophoresis, optoelectronic tweezers (OET).

\section{INTRODUCTION}

$\mathbf{T}$ HE ABILITY to manipulate individual cells is extremely attractive for cellular biologists. Cellular-level manipulation facilitates the study of single-cell behavior, such as the measurement of cellular forces [1] or cell-to-cell interactions. Single-cell manipulation can also aid in sample preparation or purification.

There are several forces that can be used to move and trap cells. Mechanical structures can be used to grip microparticles [2], [3], but it is difficult and costly to scale up mechanical devices for the parallel manipulation of single particles. Magnetic fields can impart a force [4], but the cells under manipulation must be magnetic or attached to magnetic particles. Fluid drag can transport cells [5], but it becomes difficult to achieve single-cell manipulation.

Manuscript received December 6, 2006. This work was supported in part by the Applied Biosystems, Foster City, CA, and in part by the Center for Cell Mimetic Space Exploration (CMISE), NASA University Research, Engineering and Technology Institute (URETI)

A. T. Ohta, A. Jamshidi, H.-Y. Hsu, and M. C. Wu are with the Department of Electrical Engineering and Computer Sciences and the Berkeley Sensor and Actuator Center, University of California at Berkeley, Berkley, CA 94720 USA (e-mail: aohta@eecs.berkeley.edu; arash@eecs.berkeley.edu; hsu@eecs.berkeley.edu; wu;@eecs.berkeley.edu).

P.-Y. Chiou was with the Department of Electrical Engineering and Computer Sciences and the Berkeley Sensor and Actuator Center, University of California at Berkeley, Berkeley, CA 94720 USA. He is now with the Department of Mechanical and Aerospace Engineering, University of California at Los Angeles, Los Angeles, CA 90095 USA (e-mail: pychiou@seas.ucla.edu).

H. L. Phan, S. W. Sherwood, J. M. Yang, and A. N. K. Lau are with Applied Biosystems, Foster City, CA 94404 USA (e-mail: Huan.Phan@ appliedbiosystems.com; Steven.Sherwood@appliedbiosystems. com; Joon. Yang@appliedbiosystems.com; lauan@appliedbiosystems.com).

Digital Object Identifier 10.1109/JSTQE.2007.893560
Optical forces can also be used for cell manipulation, either through radiation pressure [6] or by the force exerted by the gradient of the optical field of a highly focused laser beam, as in the classic optical tweezers trap [7]. Multiple dynamic optical tweezers traps may be created from a single input beam, through a technique known as holographic optical tweezers [8], [9]. Optical manipulation has the additional advantage of being a noninvasive noncontact method of manipulation. Although optical tweezers has been proven as a technology for cellular and inorganic particle trapping, it still has several shortcomings. In order to generate sufficient trapping forces, laser intensities of approximately $10^{5}-10^{7} \mathrm{~W} / \mathrm{cm}^{2}$ are typically used, which can adversely affect cellular behavior [10]. Cellular photodamage from high laser intensities can be reduced by using infrared lasers [11], but some effects may still remain [12]. In addition, to generate a sufficient gradient force, the laser is highly focused using objective lenses with high numerical aperture (NA). This results in a limited area over which the optical traps can be created, limiting the parallel manipulation capabilities of an optical tweezers system. This limitation becomes more restrictive for larger particles, such as mammalian cells, which typically have diameters of approximately $10 \mu \mathrm{m}$. Other types of optical trapping, such as evanescent wave trapping [13], [14], are effective over larger areas but have a difficulty in isolating a single particle from a larger population.

The electrical analog of optical tweezers is dielectrophoresis (DEP), which relies on the gradient of an electric field, rather than an optical field. Dielectrophoretic force results, when the interaction of the electric field gradient with the induced dipole of particles within the field produces a net force on the particles. Typically, microfabricated metal electrodes are used to create electric field gradients that are sufficient to trap microand nanoscale particles [15]-[17]. Dielectrophoretic manipulation is useful for a variety of functions, including cell trapping [16]-[18] and cell sorting [16], [19], [20]. However, the fixed electrode patterns limit the flexibility of individual devices, and make it difficult to isolate a particular cell from a population. Another electrical-force-based mechanism, traveling-wave DEP, relies on spatially varying voltages created by applying alternating phases to electrodes in order to transport cells [20]. The same limitations of DEP also apply to traveling-wave DEP. In order to have a dynamic single-cell control, an active DEP device is required. This has been demonstrated by the creation of dynamic DEP traps on a CMOS-circuit-based device [21]. However, the current incarnation of the device limits the resolution to $20-\mu \mathrm{m}$-diameter cells or larger, if single-cell trapping is desired. 


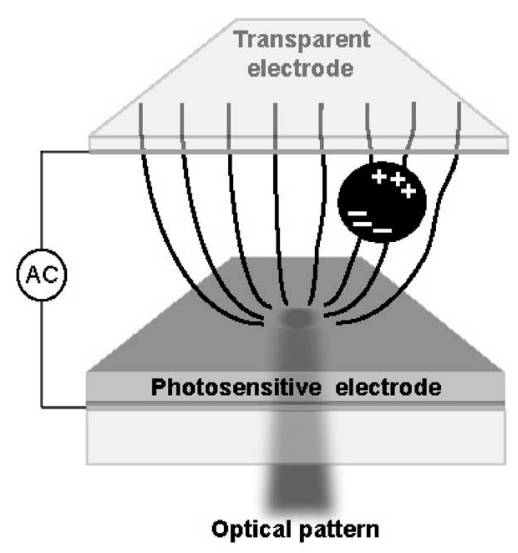

Fig. 1. Schematic of OET. The OET device is based on optically induced DEP. Illuminated areas create optically defined electrodes on the photosensitive surface, creating electric field gradients that generate a DEP force.

Another approach to single-particle manipulation combines the aspects of both optical tweezers and DEP, resulting in optically induced DEP. This technology, called optoelectronic tweezers (OET), uses a photosensitive surface to allow an optical pattern to control the electric field landscape within an OET device [22]. The resulting nonuniform electric field then generates a DEP force on particles in the OET device (Fig. 1). As optical energy is not directly used for trapping, much lower optical intensities can be used as compared to optical tweezers, in the range of $0.1-100 \mathrm{~W} / \mathrm{cm}^{2}$. These optical intensities can be achieved by a computer projector or an LED, allowing the creation of complex optical manipulation patterns [22], [23]. In addition, the optical gradient force is no longer required, relaxing the focusing requirements and allowing the use of low-NA lenses. As a result, in our current configuration, the effective manipulation of OET is 500 times larger than that of a typical optical tweezers setup. Furthermore, since the manipulation patterns are controlled optically, OET retains the flexibility and dynamic control enjoyed by optical tweezers. Unlike electrodebased DEP, OET is capable of trapping a specific single particle from a larger population.

OET manipulation has been demonstrated on a variety of microparticles including polystyrene beads [22]-[27], E. coli bacteria [28], red and white blood cells [27], HeLa cells [25], and yeast cells [29]. A variety of light sources have been used including low-power lasers, computer projectors, LEDs, and LCD displays, demonstrating the flexibility of the OET device. In addition to the advantages presented by optically controlled manipulation, OET also retains the properties of DEP. Different cells experience varying DEP forces, allowing the separation of cells based on the DEP response.

Although OET is a highly flexible and versatile device, certain limitations exist. The electric field in the OET device is created in a liquid layer between two planar electrodes [Fig. 2(a)]; thus, both the electrodes must be in contact with the liquid for the device to operate. As a result, OET cannot be integrated with other devices that do not provide a conductive planar surface.

To rectify this, we have developed another version of the OET device that retains the same functionality but combines both the electrodes of the standard OET device on a single substrate [Fig. 2(b)]. This new single-sided OET device can be used with a variety of opposing surfaces, facilitating its integration with other microdevices such as microfluidic channels. Unlike standard OET, the single-sided OET device produces an electric field that is parallel to the plane of the device; thus, we call this device lateral-field optoelectronic tweezers (LOET).

In this paper, we present the use of OET to discriminate between live and dead human white blood cells, and between Jurkat and HeLa cell lines. We also detail the design and fabrication of the first single-sided LOET device, and demonstrate the manipulation of polystyrene beads in the LOET device.

\section{OET DESIGN AND FABRICATION}

\section{A. Structure}

The OET device [Fig. 2(a)] is a two-electrode device. The upper planar electrode consists of a 100-nm-thick layer of indium tin oxide (ITO), a transparent conductive material, on a glass substrate. The lower photosensitive electrode consists of multiple featureless layers of ITO, heavily doped hydrogenated amorphous silicon (a-Si:H), and intrinsic a-Si:H, all on a glass substrate. The two electrodes are separated by spacers, creating a chamber between the electrodes that is typically $100 \mu \mathrm{m}$ in height. An aqueous solution containing the cells or particles under manipulation is introduced into the chamber between the electrodes. An electric field is created in the device by applying an ac bias across the top and bottom electrodes.

Like the OET device, the LOET device is a two-electrode device [Fig. 2(b)]. However, unlike the standard OET device, the electrodes of the LOET device are located on the same substrate. In LOET, the electrodes are laid out in an interdigitated pattern. As with OET, the electrodes are photosensitive and consist of the same layers of ITO, heavily doped a-Si:H, and intrinsic a-Si:H. In contrast to the standard OET, the electric field is created by applying an ac bias across the electrodes on the same substrate. As a result, an upper electrode is unnecessary in the LOET device. In the experiments presented here, a glass cover is typically used with the LOET device, in order to reduce the evaporation of the liquid/particle solution.

\section{B. Fabrication}

The fabrication process of the standard OET device is suitable for low-cost mass fabrication for disposable applications. This is an advantage for biological applications, as disposable devices reduce the chances of sample cross contamination. To make the OET device, ITO is sputtered onto 1-mm-thick glass wafers. The a-Si:H photosensitive electrodes are then created by plasmaenhanced chemical vapor deposition. One etch step is necessary to complete the OET device; reactive-ion etching (RIE) (90\% $\mathrm{SF}_{6}, 10 \% \mathrm{O}_{2}, 100-\mathrm{W}$ RF power) is used to remove a section of the a-Si:H to create a bias pad to apply ac bias to the ITO layer.

The fabrication of the LOET device is slightly more involved than that of the standard OET device, but is also suitable for the manufacturing of low-cost devices. The first two steps are the same as in case of the OET device. After the a-Si:H layers are 

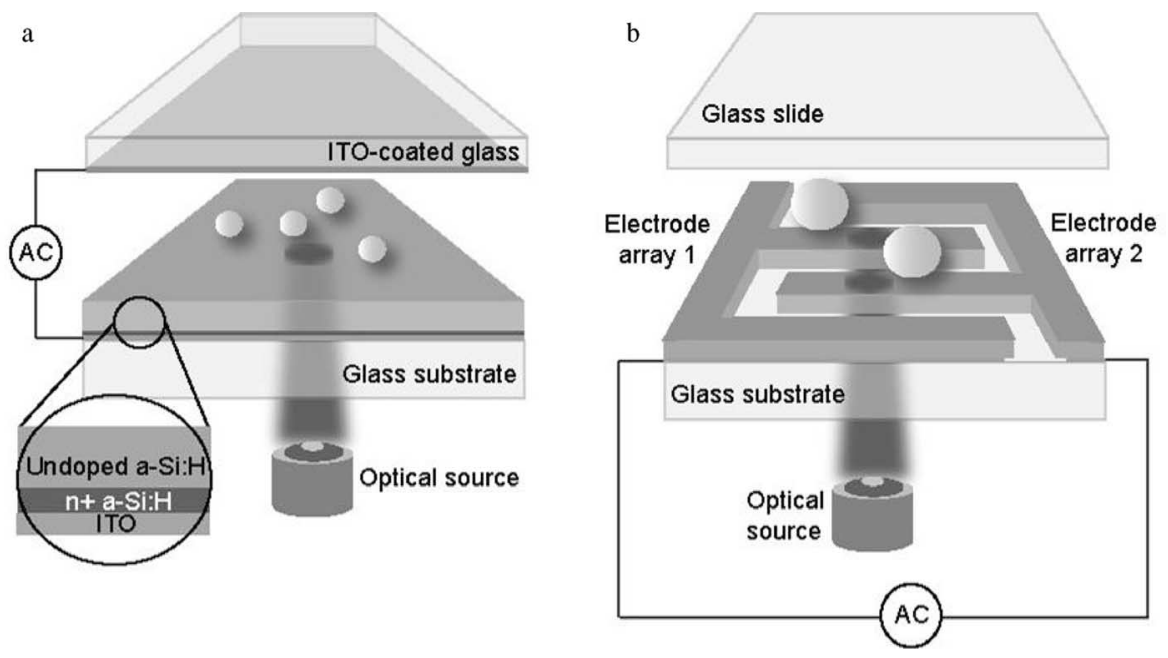

Fig. 2. Structures of the standard OET device and the LOET device. (a) Standard OET device consists of a top transparent ITO electrode and a bottom a-Si:H photosensitive electrode. A variety of optical sources such as LEDs, halogen lamps, or lasers are used to create dynamic optically defined electrodes on the photosensitive surface. (b) LOET device has both electrodes on the bottom surface, consisting of an interdigitated array of photosensitive electrodes, made of the same layers as the photosensitive electrode in the standard OET device. Optical patterns are used to control the sections of the electrode array, which are turned on.

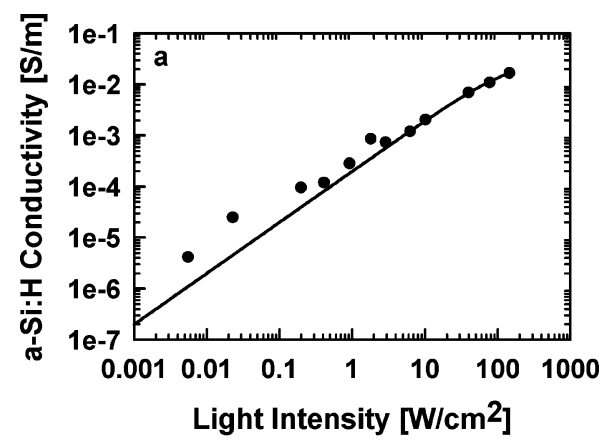

b OET device c LOET device
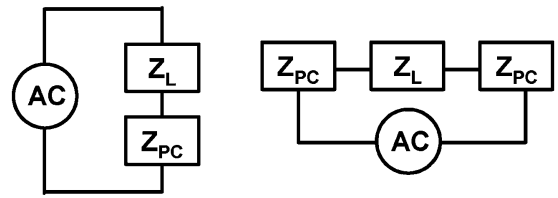

Fig. 3. (a) Measured conductivity of undoped a-Si:H as a function of light intensity at a 5-V bias. (b) Simplified equivalent circuit of the OET device. The impedance of the liquid layer is $Z_{L}$, and the impedance of the photoconductor $(\mathrm{a}-\mathrm{Si}: \mathrm{H})$ is $Z_{\mathrm{PC}}$. (c) Simplified equivalent circuit of the LOET device.

deposited, the electrode arrays are photolithographically defined and etched using RIE, stopping on the ITO layer. The ITO is then chemically etched $(38 \% \mathrm{HCl})$ to complete the formation of the interdigitated electrode arrays. Finally, a second RIE is performed to create bias pads on the ITO layer. Only two bias pads are required to address the entire LOET array, which consists of more than 450 electrode fingers.

\section{Operating Principle}

Both the standard OET and LOET devices rely on optically induced DEP to impart a force on the particles under manipulation. The dielectrophoretic force is controlled by optical patterns that are projected on the photosensitive surface of the devices.
The a-Si:H photoconductive material, which is used in the photosensitive electrodes of OET and LOET, has its conductivity modulated by the intensity of the light that is absorbed in the material [Fig. 3(a)]. Under ambient lighting conditions and a 5-V bias, the a-Si:H film has a conductivity of $0.9 \times 10^{-6} \mathrm{~S} / \mathrm{m}$. However, when illuminated with light, photogenerated charge carriers are produced in a-Si:H. As a result, the conductivity of the a$\mathrm{Si}: H$ layer increases by a few orders of magnitude to $1.7 \times 10^{-2}$ $\mathrm{S} / \mathrm{m}$ under an illumination intensity of $145 \mathrm{~W} / \mathrm{cm}^{2}$. The a-Si:H layer thus has two states: a low-conductivity dark state and a high-conductivity illuminated state. Furthermore, the transition between these two states is confined to the region under illumination, due to an ambipolar diffusion length of only $100 \mathrm{~nm}$ in amorphous silicon [30]. These two states allow the a-Si:H layer to act as a optically controlled and optically defined electrode.

The importance of the a-Si:H photoconductive layer can be seen by modeling the OET and LOET devices with a simplified equivalent circuit model [Fig. 3(b) and (c)]. In the dark state, the impedance of a-Si:H $\left(Z_{\mathrm{PC}}\right)$ is larger than the impedance of the liquid layer $\left(Z_{L}\right)$. Thus, in areas with no illumination, most of the applied ac voltage drops across the high impedance of the a-Si:H layer. However, in the illuminated areas, $Z_{\mathrm{PC}}$ becomes significantly lower, allowing a significant voltage drop to occur in the liquid layer. In this manner, an electric field gradient is set up between the illuminated and dark areas in the OET and LOET devices. However, the direction of the electric field is different in the OET and LOET devices. In the OET device, the potential difference that creates the electric field is across the top and bottom electrodes, resulting in a field that is primarily perpendicular to the surface of the OET device [Fig. 4(a)]. However, in the LOET device, the potential difference is across the interdigitated electrode fingers. When adjacent electrodes are illuminated, an electric field is formed parallel to the surface of the OET device [Fig. 4(b)]. Full 2-D control is retained in the LOET device, as the electrodes are only activated when illuminated with an optical pattern. 


\section{Simulated electric field in} standard OET

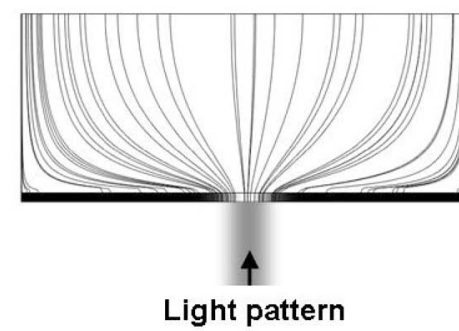

b

Simulated electric field in lateral OET

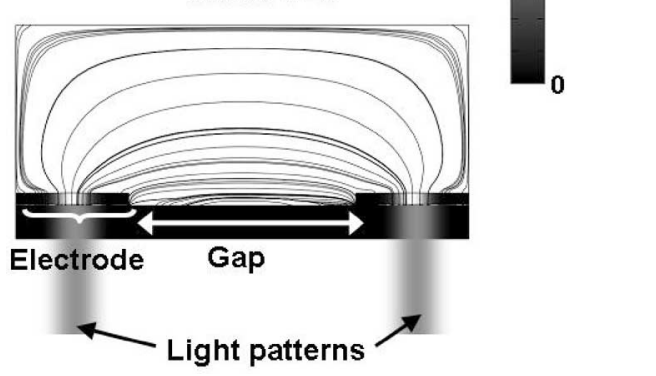

Fig. 4. Simulated electric field profiles in the standard OET and LOET devices. (a) In the standard OET device, the electric field is primarily normal to the electrode surfaces. (b) In the LOET device, the electric field is primarily parallel to the electrode surfaces.

\section{OET and LOET Forces}

Electric field gradients in the OET and LOET devices result in an optically induced DEP force. This DEP force results from the interaction of a nonuniform electric field and the induced dipole of a particle within the electric field. The forces at each end of the particle's dipole are unequal due to the nonuniform field, resulting in a net force (Fig. 1). This force can be described by

$$
F=\bar{p} \cdot \nabla \bar{E}
$$

where $\bar{p}$ is the dipole moment of the particle and $\bar{E}$ is the first term of the Taylor series expansion of the electric field [31]. If the particle is a homogeneous dielectric sphere (e.g., a polystyrene bead), then the time-averaged DEP force reduces to

$$
F_{\mathrm{DEP}}=F_{\mathrm{OET}}=2 \pi r^{3} \varepsilon_{m} \operatorname{Re}[K(\omega)] \nabla E_{\mathrm{rms}}^{2}
$$

where $r$ is the particle radius, $\varepsilon_{m}$ is the permittivity of the medium surrounding the particle, $E_{\mathrm{rms}}$ is the root-mean-square electric field strength, and $\operatorname{Re}[K(\omega)]$ is the real part of the Clausius-Mossotti factor, given by

$$
K(\omega)=\frac{\varepsilon_{p}^{*}-\varepsilon_{m}^{*}}{\varepsilon_{p}^{*}+2 \varepsilon_{m}^{*}}, \quad \varepsilon_{p}^{*}=\varepsilon_{p}-j \frac{\sigma_{p}}{\omega}, \quad \varepsilon_{m}^{*}=\varepsilon_{m}-j \frac{\sigma_{m}}{\omega}
$$

where $\varepsilon$ is the permittivity of the particle or medium (denoted by a subscript $p$ or $m), \sigma$ is the conductivity of the particle or medium, and $\omega$ is the angular frequency of the electric field [31]. The magnitude of $\operatorname{Re}[K(\omega)]$ varies with frequency, resulting in the frequency dependence of the DEP force. Positive values of $\operatorname{Re}[K(\omega)]$ result in particle attraction to electric field maxima (positive DEP, referred to here as positive OET). For negative

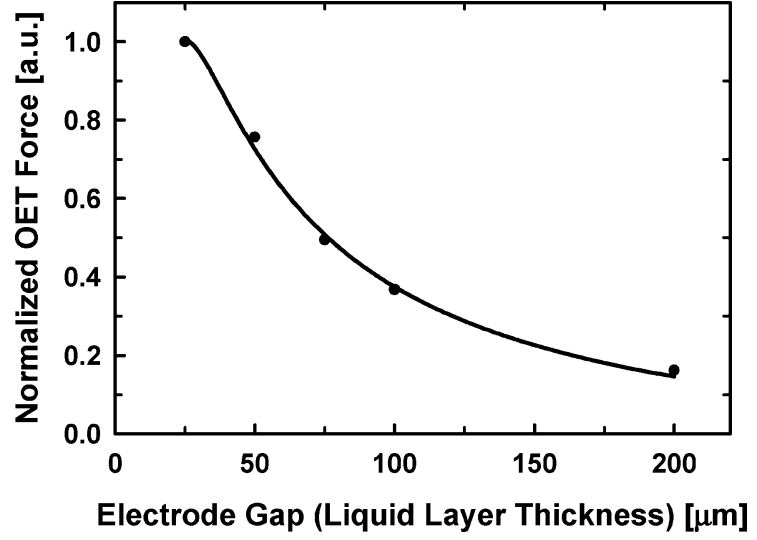

Fig. 5. Simulated OET force as a function of the gap between the OET electrodes (which also defines the thickness of the liquid layer). The bias voltage across the electrodes is held constant.

values of $\operatorname{Re}[K(\omega)]$, particles are repelled from field maxima (negative DEP, referred to here as negative OET). Applying an ac electric field, thus, allows the tuning of the type of DEP force induced on a particle, as well as negating any electrophoretic effects or particle movement due to its surface charge.

Unlike polystyrene beads, biological cells are not homogeneous. Thus, a single-shell model is typically used to determine the frequency-dependent Clausius-Mossotti factor for mammalian cells. The permittivity and conductivity of the cell membrane and interior are used to determine an effective complex permittivity that can then be used in (3). This effective permittivity is given by

$$
\varepsilon_{p}^{*}=C_{\mathrm{mem}}^{*} \frac{3 r \varepsilon_{\mathrm{int}}^{*}}{3 \varepsilon_{\mathrm{int}}^{*}+3 C_{\mathrm{mem}}^{*} r}
$$

where $\varepsilon_{\mathrm{int}}^{*}$ is the complex internal conductivity of the cell, and it is assumed that the thickness of the cell membrane $d$ is much less than the radius of the cell interior $r$ [19]. The membrane capacitance $C_{\mathrm{mem}}^{*}$ is given by

$$
C_{\mathrm{mem}}^{*}=\frac{\varepsilon_{\mathrm{mem}}}{d}-\frac{j \sigma_{\mathrm{mem}}}{d} .
$$

Many cell types are uniquely distinguishable by the real part of the Clausius-Mossotti factor. This enables the separation of different cell types using the DEP force.

\section{E. Design Considerations}

The forces induced in the standard OET device are sensitive to the gap between the top and bottom electrodes. This gap also defines the thickness of the liquid layer in the OET device. If the bias voltage across the electrodes is held constant as the electrode gap is decreased, the electric field strength in the OET device will increase. As a result, the induced OET force will also increase (Fig. 5). However, the liquid layer thickness has a practical limit. A gap that is too small may cause cells to experience significant shear stresses; thus, this gap should ideally be limited to $50 \mu \mathrm{m}$ or greater.

In contrast, as the LOET device is essentially single-sided, the force in the LOET device is insensitive to a gap between the electrode array surface and an opposing surface. Instead, the force 
induced in the LOET device is dependent on the dimensions of the electrodes including the electrode width and the spacing between the electrode fingers.

\section{EXPERIMENTAL}

\section{A. Experimental Setup}

A generalized schematic of the experimental setup of the OET and LOET devices is shown in Fig. 2. A Nikon upright microscope was used to observe the particles under manipulation. The optical pattern source consisted of one of the following: a 650-nm, 5-mW diode laser (Lasermate, Inc., LTC-6508AH), a single 625-nm LED (Lumileds, Luxeon Star/O), or a 100-W halogen lamp. The laser source was used by itself, while the LED and the halogen lamp were used in conjunction with a digital micromirror device (DMD, Texas Instruments) to produce more complex optical patterns. The optical sources were focused onto the surface of the OET or LOET device through a $10 \times, 0.3$-NA microscope objective lens (Olympus, MSPlan10).

\section{B. Cell Discrimination Using OET}

As described earlier, the DEP force is a function of the frequency-dependent electrical properties of the cells under manipulation. As different cell types exhibit dissimilar electrical properties, DEP can be used to sort between cell types, or even between widely varying cells of the same type [16], [19], [20]. This property is useful for cellular manipulation, in which heterogeneous mixtures of cells are common. We use this capability to selectively concentrate live human B cells from dead B cells, and to spatially discriminate a mixed population of Jurkat and HeLa cells.

In a live cell, the semipermeable phospholipid membrane allows a cell to maintain an ion differential between its interior and the surrounding liquid medium. In our OET experiments, cells are suspended in a low-conductivity isotonic buffer $(8.5 \%$ sucrose and $0.3 \%$ dextrose); thus, the cells have internal conductivities greater than that of the liquid. However, once a cell dies, the membrane becomes permeable to ions. The ion differential is no longer maintained, and the conductivity of the cell interior becomes the same as the surrounding liquid. This means that the Clausius-Mossotti factor is different for live and dead cells. The real part of the Clausius-Mossotti factor for a human B cell was calculated using the single-shell model of the cell, as described in (2)-(5) (Fig. 6). To determine the Clausius-Mossotti factor of dead B cells, it is assumed that the internal permittivity and conductivity of the cell is equal to that of the surrounding media, while all other parameters remain constant. The simulated results predict that, for applied frequencies greater than approximately $60 \mathrm{kHz}$, live B cells will experience a positive OET force while dead B cells will experience a negative OET force.

The difference in DEP response between live and dead B cells is used to selectively concentrate live B cells at an applied frequency of $120 \mathrm{kHz}$. The selective collection pattern is a series of broken concentric rings (Fig. 7). The pattern is created using the DMD, and illumination is provided by a $100-\mathrm{W}$ halogen lamp. As the concentric rings shrink, the live cells are focused

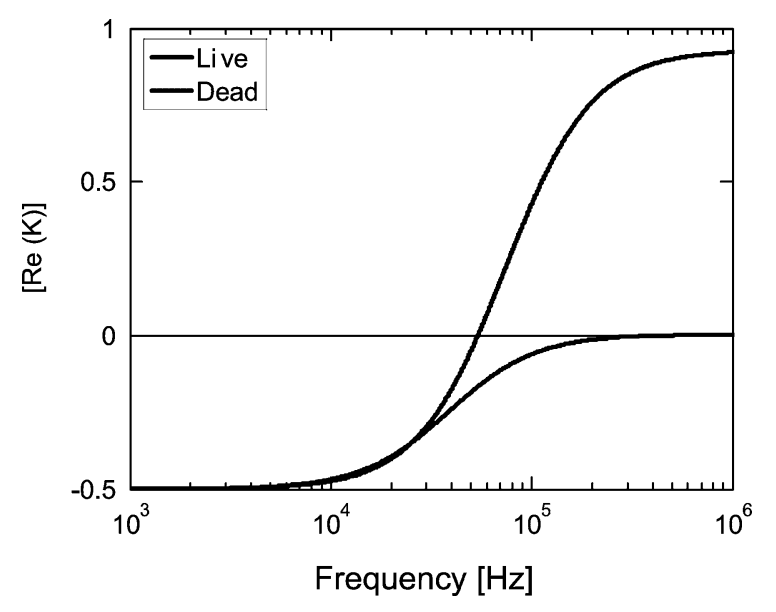

Fig. 6. Real part of the Clausius-Mossotti factor for live and dead B cells.
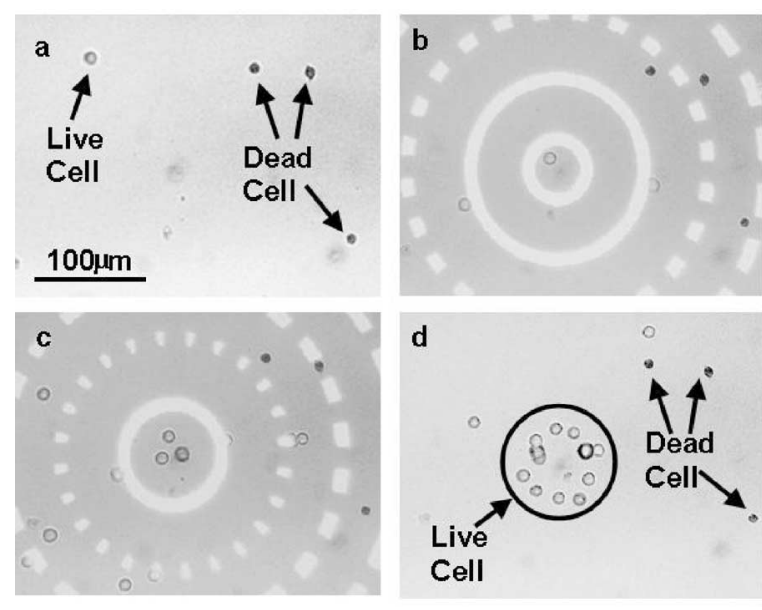

Fig. 7. Selective concentration of live B cells from dead B cells [22]. (a) Initial positions of live (clear) and dead (dark) B cells. (b) and (c) Broken concentric ring pattern is used to transport the live cells to the center of the field of view while leaving the dead cells behind. (d) Live cells have been concentrated to the central region of the optical pattern.

on the center of the pattern by positive OET. In contrast, the dead cells experience negative OET and slip through the gaps in the ring patterns. The $\mathrm{B}$ cells are suspended in isotonic solution with $0.4 \%$ Trypan blue dye. The Trypan blue dye identifies live and dead cells. Live cells exclude the dye and appear clear. The dead cells, which have a permeable membrane, absorb the dye and appear dark. The experiment is performed immediately after the addition of the Trypan blue dye, as the dye is toxic and will increase the amount of dead cells in the sample.

In addition to differentiating between live and dead cells, OET force can be used to discriminate between different cell types. This ability is demonstrated through the spatial discrimination of live Jurkat and HeLa cells using OET.

In this experiment, cultured Jurkat cells were labeled with a green fluorescent dye. The labeled Jurkat cells and cultured HeLa cells were washed with isotonic buffer, resuspended in isotonic solution, and mixed together. The concentration of the mixed solution was approximately $5 \times 10^{5}$ cells $/ \mathrm{mL}$. Culture media was added to adjust the conductivity of the cell solution 


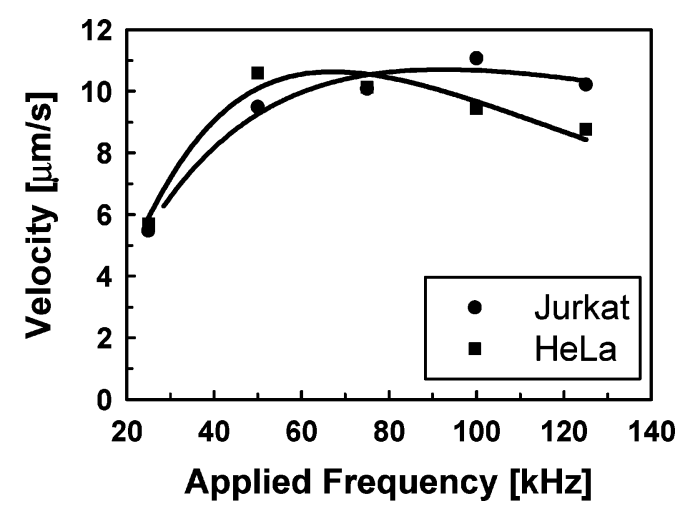

Fig. 8. OET-induced manipulation velocity of different cell types as a function of the frequency of the electric field. Cells are manipulated using a $15-\mu \mathrm{m}$-wide scanning line pattern.
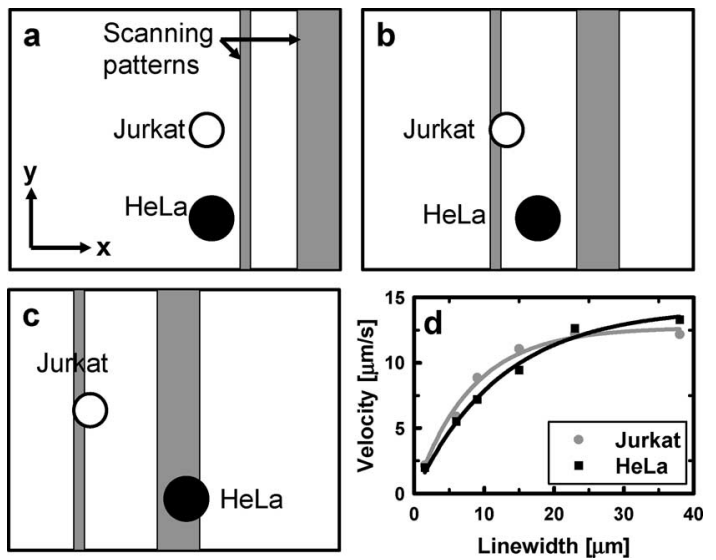

Fig. 9. Optical pattern for OET-enabled cell discrimination. (a) Cells before the lines are scanned in the negative $x$-direction. The thin leading line produces a weaker OET force than does the thicker trailing line. (b) As the pattern is scanned, the Jurkat cells have a sufficiently OET strong force to be retained by the leading line, while the HeLa cells do not experience sufficient force. (c) Trailing line provides enough force to transport the HeLa cells, while the Jurkat cells continue to be retained by the leading line, achieving spatial discrimination. (d) OET manipulation velocity of Jurkat and HeLa cells as a function of the width of the optical pattern.

to approximately $2 \mathrm{mS} / \mathrm{m}$. A $20-\mu \mathrm{L}$ aliquot of cell solution was introduced into the OET device for manipulation. The cells experienced a positive OET force and were attracted toward the optical manipulation patterns.

The maximum OET manipulation velocity of Jurkat and HeLa cells as a function of the frequency of the applied voltage was empirically determined, and is shown in Fig. 8. The manipulation velocity is proportional to the OET force. At an applied voltage of $10 \mathrm{Vpp}$ at $100 \mathrm{kHz}$, sufficient variation in the OET force exists to differentiate between the two cell types.

In order to spatially separate the HeLa and Jurkat cells, a scanning line optical pattern is used to exploit the differences in OET force on the cells. The scanning lines are produced using the DMD and an LED source, and are shown schematically in Fig. 9. A $15-\mu \mathrm{m}$-wide leading line and a $23-\mu \mathrm{m}$-wide trailing line are separated by $\sim 40 \mu \mathrm{m}$, and are simultaneously scanned at a rate of $13 \mu \mathrm{m} / \mathrm{s}$. The leading line produces a weaker OET force than does the thicker trailing line, as the manipulation

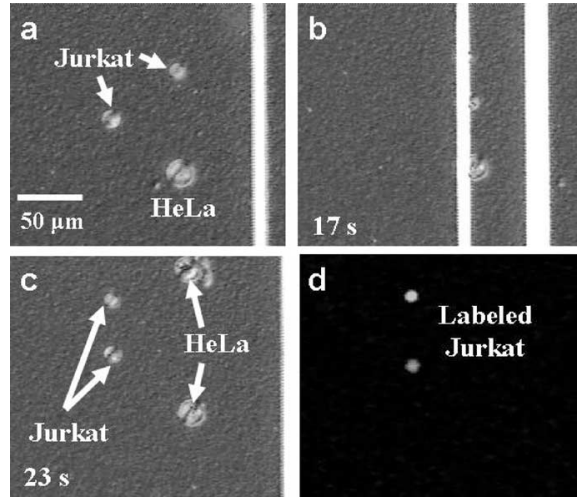

Fig. 10. OET-enabled spatial discrimination of live Jurkat and HeLa cells. (a) Initial cell positions before the optical pattern is scanned from right to left across the field of view. (b) Cells are attracted to the leading line. The HeLa cell is starting to lag the scanning line. (c) Cells showing spatial separation after the scan is completed. An additional HeLa cell has moved into the field-of-view during the scan. (d) Fluorescent image of the cells in (c), verifying that the leading cells are the fluorescent-labeled Jurkat cells.

velocity of cells exhibits a dependence on the width of the optical pattern [Fig. 9(d)]. Thus, as the two lines are scanned across the OET device, the Jurkat cells, which experience a stronger OET force, are held by the leading line. The leading line does not produce sufficient force to transport the HeLa cells against the viscous drag, which are then subsequently attracted to and transported by the trailing line. After the scan is completed, the cells retain a spatial separation equal to the spacing of the two scanning lines.

The results of the optical line scanning on a mixed population of Jurkat and HeLa cells are shown in Fig. 10. An initial group of both cell types is present [Fig. 10(a)]. As the scanning pattern moves from right to left across the field of view, the Jurkat cells are transported by the $15-\mu$ m leading line [Fig. 10(b)]. The HeLa cells are transported only slightly by the leading line pattern, and cannot maintain the velocity of the translated leading line. The $23-\mu \mathrm{m}$ trailing line then attracts and transports the HeLa cells. Scanning of the line patterns was repeated twice to achieve the desired separation between the Jurkat and HeLa cells. After the third scan, the two cell types are spatially separated [Fig. 10(c)]. A second HeLa cell is also visible, which moved into the field of view during the scanning of the optical pattern. By using fluorescent imaging, it is verified that the cells on the leading line pattern are the fluorescent-labeled Jurkat cells [Fig. 10(d)]. The unlabeled HeLa cells do not appear in this image.

\section{Microparticle Manipulation Using LOET}

In order to demonstrate that the LOET device retains the 2-D manipulation pattern capabilities of the standard OET device, initial tests were performed using polymer microparticles. Polystyrene beads with diameters of 25 and $45 \mu \mathrm{m}$ are suspended in a solution of $\mathrm{KCl}$ and deionized water, which is adjusted to attain a conductivity of $10 \mathrm{mS} / \mathrm{m}$. Approximately $20 \mu \mathrm{L}$ of the bead solution is dispensed into the LOET device.

Polystyrene bead manipulation in the LOET device was tested using a laser source. The LOET devices had electrodes that were 


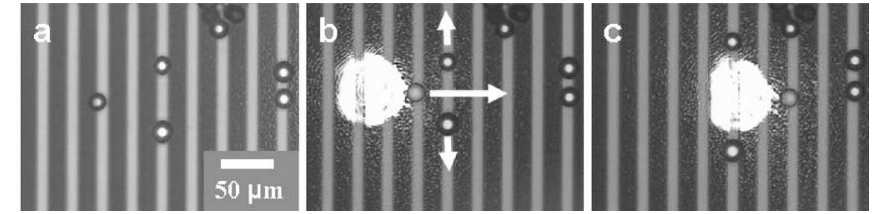

Fig. 11. Movement of the $25-\mu \mathrm{m}$-diameter polystyrene beads in the LOET device. (a) Initial positions of the beads. (b) Laser is used to transport a single bead in the transverse direction, while simultaneously moving other beads in the longitudinal directions. Arrows indicate the direction of motion. (c) Final position of the polystyrene beads.
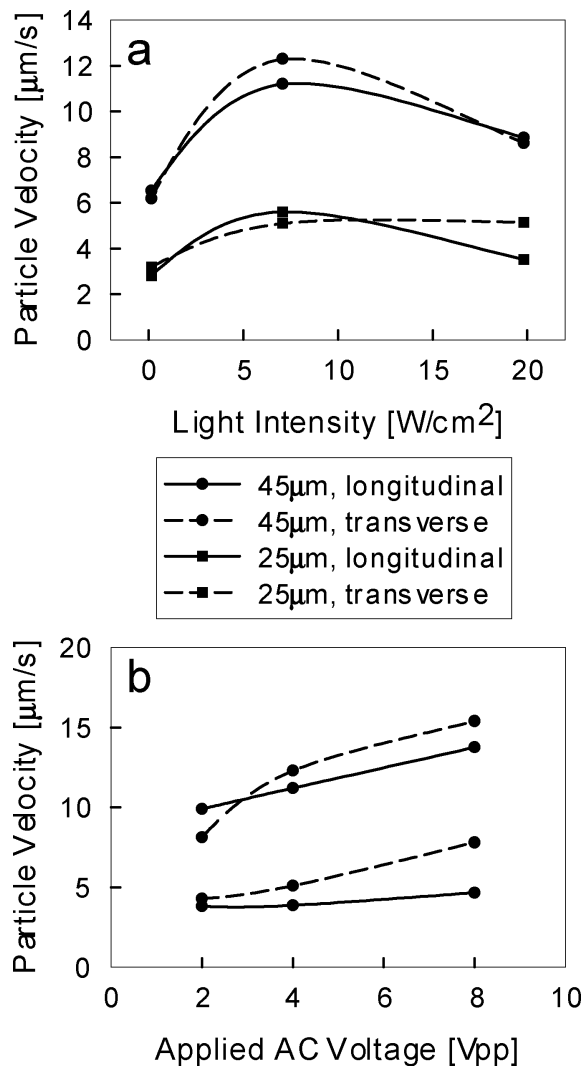

Fig. 12. Experimental induced velocities of polystyrene beads in the LOET device. Longitudinal velocity refers to movement along an electrode, while transverse velocity refers to movement across electrode fingers. (a) Particle velocity as a function of illumination pattern intensity. (b) Particle velocity as a function of applied ac voltage at a frequency of $100 \mathrm{kHz}$.

$10 \mu \mathrm{m}$ in width, with a gap of $20 \mu \mathrm{m}$ between electrode fingers. The electrode arrays were biased with an ac voltage of $4 \mathrm{Vpp}$ at $100 \mathrm{kHz}$. Both longitudinal and transverse motion of $25-\mu \mathrm{m}-$ diameter polystyrene beads was observed (Fig. 11). Longitudinal motion refers to particle movement along the length of the electrodes, and transverse motion refers to particle movement across electrode fingers.

The velocity induced in the LOET device on 25- and $45-\mu$ m-diameter polystyrene beads is shown as a function of the illumination light intensity and applied ac voltage (Fig. 12). Both the longitudinal and the transverse directions of movement have approximately the same manipulation velocities, further confirming that full 2-D motion is preserved on the LOET device.

\section{CONCLUSION}

Dynamic complex optical patterns may be used with OET to control the movement and location of many particles at once, effectively creating thousands of individual particle traps on a featureless surface. The ease and flexibility in creating these traps makes OET attractive compared to electrode-based particle manipulation technologies. Furthermore, OET enjoys some advantages over purely optical manipulation tools. The actuation intensity required to produce an OET trap is up to five orders of magnitude lower than that of an optical tweezers trap. In addition, as optical gradient forces are not used in the OET device, the optical patterns used for OET need not be highly focused, thus, allowing OET manipulation to be performed over an area 500 times larger than that in typical optical tweezers traps. Although OET is optically controlled, the OET forces still retain the same properties as in conventional DEP, allowing the use of OET force to separate between different cell types. We have demonstrated the selective concentration of live human B cells from a mixed population of live and dead B cells, as well as the spatial separation of a mixed population of Jurkat and HeLa cells.

Although OET manipulation has been demonstrated to be highly flexible, device integration is sometimes challenging as OET is a two-sided device. Thus, the integration of OET manipulation capabilities with a variety of other technologies such as microfluidic channels will be greatly facilitated by the single-sided LOET device. Here, we have outlined the design and fabrication of the LOET device, and demonstrated LOET manipulation on polystyrene beads at velocities greater than $15 \mu \mathrm{m} / \mathrm{s}$.

We envision both types of OET devices complementing each other. Together, these OET technologies provide powerful tools for the manipulation of micro- and nanoscale particles.

\section{ACKNOWLEDGMENT}

All devices discussed here were fabricated at the University of California at Berkeley (UCB) Microfabrication Laboratory. The authors would like to thank the UCB Tissue Culture Facility for providing the cells used in the experiments, and the Photonics Technology Access Program (PTAP) for providing the fast spatial light modulators.

\section{REFERENCES}

[1] B. Maier, M. Koomey, and M. P. Sheetz, "A force-dependent switch reverses type IV pilus retraction," Proc. Natl. Acad. Sci. USA, vol. 101, pp. 10961-10966, 2004.

[2] C. J. Kim, A. P. Pisano, and R. S. Muller, "Silicon-processed overhanging microgripper," J. Microelectromech. Syst., vol. 1, pp. 31-36, 1992.

[3] N. Chronis and L. P. Lee, "Electrothermally activated SU-8 microgripper for single cell manipulation in solution," J. Microelectromech. Syst., vol. 14, pp. 857-863, 2005.

[4] H. Lee, A. M. Purdon, and R. M. Westervelt, "Manipulation of biological cells using a microelectromagnet matrix," Appl. Phys. Lett., vol. 85, pp. 1063-1065, 2004.

[5] G. M. Whitesides, "The origins and the future of microfluidics," Nature, vol. 442, pp. 368-373, 2006.

[6] A. Ashkin, "Acceleration and trapping of particles by radiation pressure," Phys. Rev. Lett., vol. 24, pp. 156-159, 1970. 
[7] A. Ashkin, J. M. Dziedzic, J. E. Bjorkholm, and S. Chu, "Observation of a single-beam gradient force optical trap for dielectric particles," Opt. Lett., vol. 11, pp. 288-290, 1986.

[8] D. G. Grier, “A revolution in optical manipulation," Nature, vol. 424, pp. 810-816, 2003.

[9] P. A. Prentice, M. P. MacDonald, T. G. Frank, A. Cuschieri, G. C. Spalding, W. Sibbett, P. A. Campbell, and K. Dholakia, "Manipulation and filtration of low index particles with holographic Laguerre-Gaussian optical trap arrays," Opt. Express, vol. 12, pp. 593-600, 2004.

[10] A. Ashkin and J. M. Dziedzic, "Optical trapping and manipulation of viruses and bacteria," Science, vol. 235, pp. 1517-1520, 1987.

[11] A. Ashkin, J. M. Dziedzic, and T. Yamane, "Optical trapping and manipulation of single cells using infrared laser beams," Nature, vol. 330, pp. 769-771, 1987.

[12] K. C. Neuman, E. H. Chadd, G. F. Liou, K. Bergman, and S. M. Block, "Characterization of photodamage to Escherichia coli in optical traps," Biophys. J., vol. 77, pp. 2856-2863, 1999.

[13] M. Gu, J. B. Haumonte, Y. Micheau, J. W. M. Chon, and X. S. Gan, "Laser trapping and manipulation under focused evanescent wave illumination," Appl. Phys. Lett., vol. 84, pp. 4236-4238, 2004.

[14] V. Garces-Chavez, K. Dholakia, and G. C. Spalding, "Extended-area optically induced organization of microparticies on a surface," Appl. Phys. Lett., vol. 86, pp. 031106-01-031106-03, 2005.

[15] M. P. Hughes, "AC electrokinetics: Applications for nanotechnology," Nanotechnology, vol. 11, pp. 124-132, 2000.

[16] P. R. C. Gascoyne and J. V. Vykoukal, "Dielectrophoresis-based sample handling in general-purpose programmable diagnostic instruments," Proc. IEEE, vol. 92, no. 1, pp. 22-42, Jan. 2004.

[17] J. Voldman, "Electrical forces for microscale cell manipulation," Аnnu. Rev. Biomed. Eng., vol. 8, pp. 425-454, 2006.

[18] D. S. Gray, J. L. Tan, J. Voldman, and C. S. Chen, "Dielectrophoretic registration of living cells to a microelectrode array," Biosens. Bioelectron., vol. 19, pp. 771-780, 2004.

[19] P. R. C. Gascoyne, X.-B. Wang, H. Ying, and F. F. Becker, "Dielectrophoretic separation of cancer cells from blood," IEEE Trans. Ind. Appl., vol. 33, no. 3, pp. 670-678, May/Jun. 1997.

[20] R. Pethig, M. S. Talary, and R. S. Lee, "Enhancing traveling-wave dielectrophoresis with signal superposition," IEEE Eng. Med. Biol. Mag., vol. 22, no. 6, pp. 43-50, Nov./Dec. 2003.

[21] A. B. Fuchs, A. Romani, D. Freida, G. Medoro, M. Abonnenc, L. Altomare, I. Chartier, D. Guergour, C. Villiers, P. N. Marche, M. Tartagni, R. Guerrieri, F. Chatelain, and N. Manaresi, "Electronic sorting and recovery of single live cells from microlitre sized samples," Lab Chip, vol. 6, pp. 121-126, 2006.

[22] P. Y. Chiou, A. T. Ohta, and M. C. Wu, "Massively parallel manipulation of single cells and microparticles using optical images," Nature, vol. 436, pp. 370-372, 2005.

[23] A. T. Ohta, P. Y. Chiou, and M. C. Wu, "Dynamic DMD-driven optoelectronic tweezers for microscopic particle manipulation," in Proc. Conf. Lasers Electro-Opt., vol. 1, San Francisco, CA, May 16-21, 2004, p. 3.

[24] P. Y. Chiou, Z. Chang, and M. C. Wu, "A novel optoelectronic tweezer using light induced dielectrophoresis," in Proc. IEEE/LEOS Int. Conf. Opt. MEMS Their Appl., Kona, HI, Aug. 18-21, 2003, pp. 8-9.

[25] P. Y. Chiou, A. T. Ohta, and M. C. Wu, "Continuous optical sorting of HeLa cells and microparticles using optoelectronic tweezers," in Proc. IEEE/LEOS Int. Conf. Opt. MEMS Their Appl. Oulu, Finland, Aug. 1-4, 2005, pp. 83-84.

[26] S. L. Neale, M. Mazilu, M. P. MacDonald, J. I. B. Wilson, K. Dholakia, and T. F. Krauss, "Size resolution with light induced dielectrophoresis (LIDEP)," Proc. SPIE, vol. 6326, p. 632618, 2006.

[27] A. T. Ohta, P. Y. Chiou, T. H. Han, J. C. Liao, U. Bhardwaj, E. R. B. McCabe, F. Yu, R. Sun, and M. C. Wu, "Dynamic cell and microparticle control via optoelectronic tweezers," J. Microelectromech. Syst., to be published.

[28] P. Y. Chiou, W. Wong, J. C. Liao, and M. C. Wu, "Cell addressing and trapping using novel optoelectronic tweezers," in Proc. 17th IEEE Int. Conf. Micro Electro Mech. Syst., Maastricht, The Netherlands, 2004, pp. 21-24.

[29] Y.-S. Lu, Y.-P. Huang, J. A. Yeh, C. Lee, and Y.-H. Chang, "Controllability of non-contact cell manipulation by image dielectrophoresis (iDEP)," Opt. Quantum Electron., vol. 37, pp. 1385-1395, 2005.

[30] R. Schwarz, F. Wang, and M. Reissner, "Fermi level dependence of the ambipolar diffusion length in silicon thin film transistors," Appl. Phys. Lett., vol. 63, pp. 1083-1085, 1993.

[31] T. B. Jones, Electromechanics of Particles.. Cambridge, U.K.: Cambridge Univ. Press, 1995.

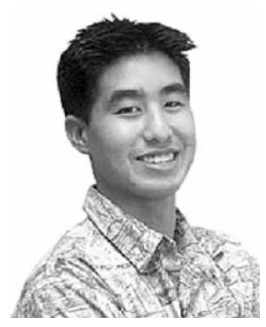

Aaron T. Ohta (S'99) received the B.S. degree from the University of Hawaii at Manoa, Honolulu, in 2003, and the M.S. degree from the University of California at Los Angeles, Los Angeles, in 2003 and 2004 , respectively, in electrical engineering. He is currently working toward the Ph.D. degree in electrical engineering at the University of California at Berkeley, Berkeley.

His research interests include microelectromechanical systems (MEMS) and bioMEMS.

Mr. Ohta is the recipient of the National Science Foundation Graduate Research Fellowship.

Pei-Yu Chiou received the B.S. degree in mechanical engineering from the National Taiwan University, Taipei, Taiwan, in 1988, the M.S. degree in electrical engineering from the University of California at Los Angeles (ULCA), Los Angeles, in 2004, and the Ph.D. degree in electrical engineering and computer sciences from the University of California at Berkeley, Berkeley, in 2005.

Since 2006, he has been a Faculty Member in the Department of Mechanical and Aerospace Engineering, UCLA. His research interests include biomicroelectromechanical systems and biophotonics.

Huan L. Phan received the Ph.D. degree molecular biology from the University of California Los Angeles (UCLA), Los Angeles, in 1995.

From 1995-2000, he was a Postdoctoral Fellow and a Scientist at Sugen, Inc., Redwood City, CA, where he identified novel protein targets for the Src tyrosine kinase. From 2000-2005, he was a Senior Scientist at Caliper Life Sciences, Mountain View, CA, where he developed microfluidic electrophoresis assays for DNA, RNA, and protein separation. He is currently a Staff Scientist at Applied Biosystems, Foster City, CA. His current interests include emerging technologies for biomedical assays and devices.

Steven W. Sherwood, photograph and biography not available at the time of publication.

Joon M. Yang received the B.S., M.S., and Ph.D. degrees in mechanical engineering from Seoul National University, Seoul, Korea, in 1989, 1991, and 1996, respectively.

From 1996 to 1999, he was a Postdoctoral Fellow with the University of California at Los Angeles, Los Angeles. Since then, he has been in the biotech industry for over seven years including Nanogen, Inc., San Diego, CA, Genoptix, Inc., Carlsbad, CA, and Applied Biosystems, Foster City, CA. He is currently a Senior Microfluidics Engineer at Applied Biosystems. He has authored or coauthored many papers published in various journals and conference proceedings. His research interests include microfluidics, and biomedical devices and instrumentation.

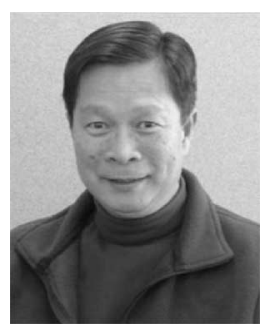

Aldrich N. K. Lau received the Ph.D. degree in organic chemistry from the University of Nebraska, Lincoln, in 1980.

From 1981 to 1983 , he was a Postdoctoral Fellow with the University of Minnesota, Minneapolis, studying electrochemically active polymers for controlled release of biomedical molecules. From 1983 to 1999 , he was a Materials Scientist at Raychem Corporation, Menlo Park, CA, working with adhesives, polymer synthesis, microencapsulation, and surface modification. He joined Applied Biosystems, Foster City, CA, in 1999, where he is currently a Principal Scientist. His current research interests include polymeric materials for DNA sequencing and surface chemistry for bioassay applications. He is the author of three book chapters, 13 journal articles, and nine papers in conference proceedings. He is the holder of 22 granted patents and 27 pending U.S. patents. 


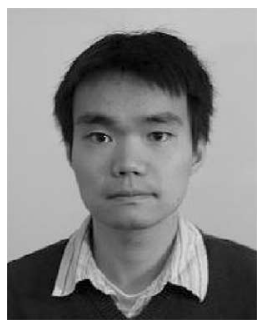

Hsan-Yin Hsu received the B.S. degree in electrical engineering from Purdue University, West Lafayette, IN, in 2005. He is currently working toward the M.S. degree in electrical engineering at the University of California at Berkeley, Berkeley.

His research interests include microelectromechanical systems, microfluidics, and optical systems for biomedicine application.

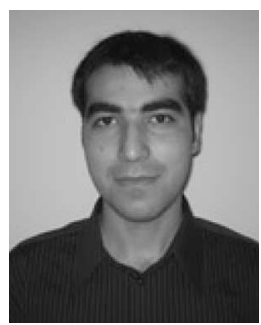

Arash Jamshidi received the B.S. degree in electrical engineering from Simon Fraser University, Vancouver, Canada, in May 2005.

He is currently a Graduate Student Researcher at the University of California at Berkeley, Berkeley, working on OET applications in the nanoscale as a part of the Integrated Photonics Laboratory Research Group. His research interests include nanotechnology, optoelectronics, bioMEMS.

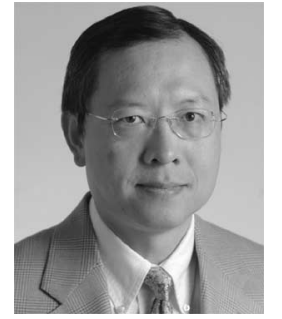

Ming C. Wu (S'82-M'83-SM'00-F'02) received the B.S. degree in electrical engineering from $\mathrm{Na}-$ tional Taiwan University, Taipei, Taiwan, in 1983, and the M.S. and Ph.D. degrees in electrical engineering and computer sciences from the University of California at Berkeley (UCB), Berkeley, in 1985 and 1988 , respectively.

From 1988 to 1992, he was a member of the Technical Staff at AT\&T Bell Laboratories, Murray Hill,

NJ. From 1992 to 2004, he was a Professor in the Department of Electrical Engineering with the University of California at Los Angeles, Los Angeles, where he was also the Vice Chair for Industrial Affiliate Program and the Director of Nanoelectronics Research Facility. In 2004, he joined the UCB, where he is currently a Professor of electrical engineering and computer sciences and a Co-Director of Berkeley Sensor and Actuator Center. His research interests include optical microelectromechanical systems, high-speed semiconductor optoelectronics, nanophotonics, and biophotonics. He has authored six book chapters, over 135 journal papers, and 280 papers in conference proceedings. He is the holder of 14 U.S patents.

Prof. Wu is a member of the Optical Society of America. From 1992 to 1997, he was a Packard Foundation Fellow. Since 1996, he has been the founding Co-Chair of IEEE/LEOS Summer Topical Meeting on Optical MEMS. He has participated in the program committees of many technical conferences. He is the Guest Editor of two special issues of IEEE JOURNAL ON OPTICAL MEMS. 